\title{
POWER SHARING IN MEDICAL CONSULTATIONS
}

\author{
Ayesha Junaid, Muhammad Shaban Rafi, Najm Us Saqib Khan*, Junaid Sarfraz Khan** \\ University of Management \& Technology, Lahore Pakistan, *Naval Headquarters, Islamabad Pakistan, ${ }^{* *}$ National University of Medical \\ Sciences (NUMS) Rawalpindi Pakistan,
}

\begin{abstract}
Objective: To examine the management of power by doctors in medical consultations. The power is defined here as a dialogic, egalitarian, and patient-centered.

Study Design: Qualitative study.

Place and Duration of Study: Out-patient departments of Mayo Hospital, Lahore, Pakistan, from Nov 2019 for two weeks.

Methodology: The data were collected through in-depth interviews and observations from outpatient departments of Mayo Hospital. Bourdieu's Social Practice Theory and Fairclough's theory of Power and Language were used as a theoretical framework in the community of practice, for the interpretation of the qualitative data sets.

Results: The interpretations of relational power by doctors and patients surface three themes: Power, Power and Solidarity, and Solidarity. Although power-sharing is the modern rhetoric, it is hardly conceptualized in the selected hospital.

Conclusion: With a proliferation of patient-centered approach of the medical profession, power-sharing with patients might perpetuate dissatisfaction among the participants.
\end{abstract}

Keywords: Critical discourse analysis, Doxa, Habitus, Medical consultation, Power, Solidarity.

This is an Open Access article distributed under the terms of the Creative Commons Attribution License (http://creativecommons.org/licenses/by/4.0), which permits unrestricted use, distribution, and reproduction in any medium, provided the original work is properly cited.

\section{INTRODUCTION}

Power is considered as a relational and an influential process that is co-constructed in an interaction. Power is seen as a facilitator or restriction in interactions ${ }^{1}$. Power comes into existence when particular strategies are used during an interaction, e.g., doctors use medical jargon and habits such as topic control and turn snatching. He may use power to share their knowledge and include patients in decision-making process. These dispositions become a subconscious part of the doctors' personality due to their training as part of being professionals. The doctors possess such power as they have reliable knowledge on which their patients are dependant $^{2}$. The discourse between the doctors and patients is asymmetrical, which can be a source of mistrust on the patients' part. In a study, suggestions were given to overcome barriers to improve doctor-patient interaction from the doctor's pers-

Correspondence: Dr Ayesha Junaid, PHD Scholar, University of Management \& Technology, Lahore Pakistan

Received: 01 Oct 2020; revised received: 10 Oct 2020; accepted: 14 Oct 2020 pective. Power interwoven in language is a tool of meaning-making system ${ }^{3}$. Meaning is co-constructed in an encounter through which power is exerted 4 .

Fairclough's theory of Power and Language, along with Bourdieu's Social Practice Theory ${ }^{5}$, were used as a research framework. Habitus is a set of learned dispositions, which force an individual to act, react and respond in a certain way. Doctor's training and profession makes him an embodiment of a set of dispositions learned unconsciously. The concept of doxa furthers the notion of habitus. Doxa makes sense of how doctors internalize a set of conventions, knowledge, beliefs, and an institution's culture without knowing that they are doing so. Although this process is subconscious, it influences the thinking patterns of doctors.

Critical Discourse Analysis (CDA) uses language, discursive practices, and discursive events to analyze the data on multiple levels of description, interpretation, and explanation. The two theories in integration, facilitated in analyzing doctor-patient interactions, doctors' language and 
discourse, and their dispositions and their perceptions about power-sharing with patients.

The research in doctor-patient interaction is essential, especially when there is a demand for dialogic and egalitarian patient-focused care.This research aims to examine the dominant discourse, whether doctors share power with the patients or not in medical consultations in the age of increasing patient-centered approach towards patient care $^{7,8}$. To the researchers' knowledge, this area has been unexplored in Pakistan. This study asked the following questions: 1-Do power relations exist in doctor-patient interactions, and how do doctors deal with these power relations?. 2-Does a difference exist between doctors' perceptions about power and in actual clinical practices?

The study was conducted in Mayo Hospital, Lahore, Pakistan, in November 2019. A purposive sampling technique was used to recruit ten doctors as participants. The inclusion criteria for selecting doctors were set as; having at least five years of experience in teaching and training from the departments of Surgery, Paediatrics, Dermatology, Dentistry, and Psychiatry.

The data were collected over two weeks. Ten doctors and ten patients were interviewed and later observed in the clinics. The data were collected through semi-structured interviews that lasted for about half an hour to one hour, in the Urdu language. The same set of questions was asked to the doctors and patients. The researchers asked the doctors two significant questions; Are there power relations when interacting with your patients? If yes, how do you deal with that? The researchers asked some follow-up and probing questions if the respondents did not come up with clear answers. The observations were obtained on a checklist developed by the researchers consisting of sixteen points. These points were used to check the doctors' behaviors, as well as their communicative strategies that were recorded in the form of numbers and percentages. A total of 100 consultations were observed. The principal researcher observed the consultation process as a quiet observer.
The interviews were recorded, transcribed, and translated in English. The data from observations and interviews were analyzed qualitatively. The consultation sessions were observed focusing on how the participants handled/mishandled the language by using politeness strategies, verbal and non-verbal cues such as eye contact, smiles, and gestures. An examination of the words used by the doctors and the patients during medical interaction helped to determine the power relations between them. The participants' verbal and non-verbal features of communication skills (nodding, smile, and handshake) were noted to understand the meaning-making process.

The checklist provided a subjective assessment from the observations, and the researchers created a link between the interviews and the observations through analyzing the linguistic features of the dataset by using CDA and social theory.

Ethics approval was granted for the Ethical Reviewer Committee of the Department of Linguistics and Communications, University of Management and Technology, Lahore (ERC: 1693). The researchers ensured the confidentiality and unauthorized use of the data. For this purpose, the researchers refrained from using both the respondents' names and the hospital where the study took place.

\section{RESULTS}

Results from interviews were formulated by using direct quotations from the respondents. The questions asked in the interviews about power did not make sense to the respondents at first. For example, the respondents repeated back the word power for clarification. However, when given time to reflect on the question, they came up with various responses to formulate themes for the data. After repeatedly reading the transcribed verbatim, the dataset was coded for commonly emerging themes by the researchers. Patterns were analyzed by contrasting, comparing, and integrating the items with other items through triangulation of observational data and interview data. The emerging themes were: Power 
Use in Medical Consultations, Power Waning and Use of Solidarity, and Solidarity is Power.

\section{Power Use in Medical Consultations}

Some of the respondent doctors and patients perceived that doctors are the sole managers in doctor-patient interactions. They believed that the power dynamics between the doctors and patients were not only explicit but also unambiguous (see table-I). The statements by the doctors revealed that their grasp on knowledge and their patients' lack of it empowered them. Stress ordered phrases such as obviously, I have to, we know, this is so obvious, inthe doctors' rhetoric, show that they were aware of their authority. Clearly, the doctors had distinguished themselves from the patients by using in-group identity markers such as "us" and "we".

In contrast, doctors used "them" and "they" for the patients, which constitute the other group. The doctors mostly used declarative and assertive speech acts to assert their control. They did not fully listen to the patients' problems and gave prescriptions based on their general physical conditions.

Most of the doctors gave interviews in the English language, which is considered the language of prestige. Those who started their interview in Urdu, regularly code switched to English to show their top hierarchy. The personal pronoun "I" was used repeatedly to assert their assertiveness. The use of deixis showed the power differentials in the data obtained. The doctors belonging to this category mostly used distal deixis such as; "that", "over there", "then".

On the other hand, the patients used proximal deixis such as "here", "now", and "this" to show closeness and submissiveness. Deixis signalled temporal and spatial distance both by the doctors and by patients. Some doctors replied to the researcher's question by asking the question in response: "Why else would the patients otherwise come to us if they do not trust us in decision-making?".
Seven out of ten $(70 \%)$ patients believed that doctors have all the authority over them. This was evident in their rhetoric as they said, "Where else to go,what choice do we have?", "Yes, doctors have power". The patients' submissive answers revealed that they had no other choice but to trust the doctors. This was evident from their statements: "What do you think other choices we have?".

The doctors stated that they should not take advantage of their position as the patients believed and trusted them and they do not want to impair their patients' trust. However, they subconsciously exerted their power by; making decisions for the patients ("We have to assert our decision for their good"). The respondents adhering to the power theme did not believe in sharing their knowledge with patients.

The actual observations of many doctorpatient encounters revealed power dynamics in doctors' clinical practice. In one of the encounters between the dentist and the patient, which lasted for 10 minutes, it was noted that both the dentist and the patient followed some of the politeness strategies to save each other's face. The conversation between the two parties stayed smooth, as both of them knew their places. The patients knew that the doctors are power figures and following their instructions benefitted them. It was observed that the patients were submissive in those interactions. Most of them used words that show supremacy for doctors. These words include "sahib" or "sir". This linguistic tendency of patients indicated that they recognised their status. They listened to the doctors patiently. Through their habitus, doxa, and community of practice, the doctors also asserted their power in making decisions for the patients, using jargons and not including patients in decision-making. They did not keep eye contact with patients. They wrote prescriptions when the patient spoke and they prescribed tests and medicines without giving the rationale of why they were doing so. They hardly used any interpersonal language to break the ice. The patient remained uneasy due to 
the lack of exhibiting effective communication skills.

The patients interrupted the doctors while explaining a particular aspect. Doctors too entertained the other interruptions such as use of mobile and another person's entrance in room. Doctors used jargon as part of their habitus but explained if they were asked. The tone of the doctors stayed firm during the interactions. They mostly used declarative and assertive speech acts: take medicine on time, and open your mouth. The doctors did not encourage any social interaction. Rather they discouraged it. The paralinguistic features such as using silence and the doctors' use of gaze on irrelevant questions by the patients showed that, at times, the doctors became irritated as the patients did not or had not followed the instructions or were ill-disciplined. Another feature that restrained their interactions was over workload and lack of time that caused rushed interactions.

\section{Power Waning and Use of Solidarity}

Considering this theme, three out often doctors $(30 \%)$ and six out of ten $(60 \%)$ of the patients thought that doctors' power was decreasing. The doctors showed reluctance and helplessness in giving away their power. One of the doctors was of the view that: "The whole business is patientoriented. So yes, we deliberately give away our power". The loss of doctors' power and its relative gain by patients has resulted in a general feeling of disrespect and mistrust for each other. For example, one of the doctors said: "They want us to do what they want sometimes their choice may not be very preferable in our opinion. This leads to a clash of opinions".

The doctors and the patients believed that doctors' power was waning over time. The observations show that the doctors sometimes gave rationale of the prescribed medicine and tests. They also explained to the patients their disease in easy language. They made sure by repetitions that the patients had understood them well. They imparted their knowledge in trying to explain to the patients how to cure their disease. They also frequently code switched to Punjabi if they felt that the patients could not understand Urdu.

There were incidences in the observed data where it was evident that how the doctors changed their behaviour when someone told them that they had come from some reference. It was the power from above that had forced the doctors to give away their institutional power (table-I). The paralinguistic features such as silence and no eye contact at the beginning of this encounter showed the doctor's reluctance to continue interaction with the patient. However, the patient's introduction of reference: "Mujhay Dr. X nay bhaijahai." (Dr. X has sent me), changed the whole scenario, as seen in table-II. It intentionally did not state something and purposefully made insinuations. It was the patient who was exerting his power over the doctor by being assertive. He got this power as he had come from the reference of a figure of more or equal power than the doctor himself/herself.

Table-I: Transcribed interviews.

\begin{tabular}{|c|c|}
\hline DOCTOR & PATIENTS \\
\hline $\begin{array}{l}\text { 1. Obviously...patients come to us for examination. Some of } \\
\text { them believe that we will cure them fully without keeping in } \\
\text { mind our limitations. Sometimes, I try to empower the patients } \\
\text { by sharing information with them, so they can best decide for } \\
\text { themselves what to do next but this does not work always. I } \\
\text { have to assert my decision for their good. }\end{array}$ & $\begin{array}{l}\text { We believe in the doctors. What do you think other } \\
\text { choice we have? We don't know about what's } \\
\text { happening with our bodies, so we have to rely on them. } \\
\text { They are our saviours and we give them the power to } \\
\text { do what is right for us. That's all we can do as we are } \\
\text { helpless. }\end{array}$ \\
\hline $\begin{array}{l}\text { Yet another doctor went on to say that: } \\
\text { This is so obvious... we have the knowledge about their diseases } \\
\text { and the treatment that has to be provided, so yes, this } \\
\text { knowledge empowers us. I mean if I am a heart surgeon and I } \\
\text { know very well about my area. However, if I myself or my } \\
\text { family members need treatment from other specialists, they }\end{array}$ & $\begin{array}{l}\text { We sit and wait for our turn, for hours. We see that } \\
\text { mostly the people who came after us are treated first } \\
\text { and these people are also treated nicely. I think it is } \\
\text { unfair. When it is our turn, the doctor on duty whom we } \\
\text { came to meet from far away, is gone as his/her duty } \\
\text { hours were over or they are on their lunch and tea }\end{array}$ \\
\hline
\end{tabular}


obviously will have power over me.

One of the doctors said:

People entrust us with their lives. The society views things like this they haven't got much choice I suppose. So yes, it's their need that has empowered us. Anyway, in any relationship there are always power relations and in doctor patient relationship, we own the power, as it is inherent but we need to respect it and recognize it. It is taken for granted by many doctor, which is wrong and being irresponsible. It is important to recognize and accept this fact for the betterment of the patients.

Why else do you think are riots between health facility members and the patients or their relatives. They need to respect our decisions. We are humans and we can make an error that is sad, but they have no choice other than that to comply with our decisions. Of course, we lament if something goes wrong from our side but we at least try to set things right. What else options do they have, but to trust our knowledge and instincts ... and that gives us enormous power. Patients put their trust in us because they have little or no knowledge of their diseases.

Yes, in patient-doctor interaction, there are power imbalances which cannot be denied. Denying this would be hiding from the reality. A doctor has the power to make decisions for the treatment of patients. Why else would the patients otherwise come to us if they do not trust us in decision making? We are powerful. At least we need this little liberty. I would personally dislike it if the patients or their relatives interfere with my decisions.

DOCTORS
1. I do not get time even to go to the washroom. Life is so busy
in the hospital. Patients keep on entering one after another and
at times, there is a huge crowd around my table... sometimes I
think that I cannot take it anymore.... We are used to seeing so
many patients per day that now after so many years of
experience, they seem to us more of the objects than human
beings. We have to control our emotions or we won't succeed in
giving consultation to even a single one.

2. Oh, what are you talking about the power dynamics in patient-doctor interactions are rare these days. Patients are aware of what they want. The whole business is patient oriented and we have to be extra good to save our name in this tough world. So yes, we deliberately give away our power. Sometimes it becomes a real problem it really depends on the kind of patient we are dealing with. There are patients who would endlessly argue with us regarding their treatment plan. They want us to do what they want...sometimes their choice may not be very preferable in our opinion. This leads to clash of opinions. Some patients agree to us after long explanations but there are others who keep on arguing... it wastes a lot of our time as we have to see other patients waiting outside.

At times we face patients who are very demanding and bully. This can be very frustrating as they are hard to satisfy. break. I think it is in doctor's power to control this injustice.

It depends on the kind of person you are dealing with. Some doctors treat us well but mostly we are left unheard. They give us a long list of tests which are really costly and are not included in our insurance. Some of the doctors give us free medicine too as it is a public hospital, but coming from far away costs us a lot.

We come from far away to the cities as there's hardly any good healthcare facility available in the villages. Yes, we believe that doctors are powerful. We don't have any choice.

1. We have to pay so many bills the doctors'fees are so huge. We have to assert what we want. It is in a way we are buying health services from them and we do not want to compromise on health.

I don't know I personally have very bad experiences with the doctors. I avoid visiting them unless absolutely necessary. It is such a shame; doctors don't have much time for the patients the earlier concept of a doctor as a messiah is diminishing.

2. How can I trust my doctor when he doesn't tell me what is going wrong with me. I mean, I have a full right to know so I can decide for myself what to do next....

3. Personally I would prefer going to a private doctor who listens to me fully. I do not like rude doctors. There's hardly a smile on their faces and they are so jittery. I hate it that even if they have to talk, they would use jargons. I always ask for clarification as my medical 
knowledge is low. Some of them are kind enough and they answer to us in our language if possible but experienced doctorsare hardly of that kind.

4. Usually I take two opinions from different doctors if the problem is severe. I cannot take risk...these doctors are mostly overburdened and do not have time to listen to us in detail.

\section{DOCTOR}

1. What Power? Oh no, I don't think so. I think it is their right to get the service properly and we are here to provide that happily and they trust us for that. It is our moral duty not to impair their trust.

2. Most of the patients are really very intelligent. They ask you brilliant questions. They want to learn about themselves. I think we are here to guide them, so yes, we should provide them essen-tial basic knowledge whenever possible. It is good that people are becoming aware of the importance of knowledge these days....yes, even the illiterates from rural areas can ask you pretty surprising questions so I let them question. I let them be empowered.

3. They (patients) are here to get their medical problem solved and we are here to provide them so... why they would bother about power struggle? There is no competition going on! No there is no power differential in this relationship. It is a relation of mutual trust.

Table-II: Observations.

2i: Example of Power

Patient: Salam Sahib (Greetings)

Dentist: Bibi, apni bari da intizar karo (Bibi, wait for your turn).

The patient goes and sits in the corner.

Dentist (to the patient on dentist chair): Baba Ji, mai nay aap say kaha hai keh pura mu kholain. Daant nahi saaf kertay kia. Aap kay mu mai ulcer hogia hai. Mai aapko dawa daita hon jis say sojhan kam ho jai gi. Phir tusi ik haftay baad aa kay mainu waikhain. Mai aithay haftay day din honda hoon. (I have told you to open your mouth wide. Don't you brush your teeth? There's ulcer in your mouth. I'll give you a medicine that will cure your swelling. You can check back on me after a week. I'm available thrice a week here).

Patient: Sahib, takleef kamho jai gi? (Will pain be reduced)?

Dentist (keeping eye contact): Inshallah dawa hospital kay store say lay lain... pehli dawa din mai do baar subha sham khain. Dusri dawa say din mai do martaba gharary karain. Aglay haftay inshallah sojhan kam ho gi toh mujhay aap ki daar nikal nihogi. Dawa waqt pay lain. Inshallah afaqa hoga. (Take medicine from hospital store. Take first medicine twice a day; morning and evening. Gargle twice a day. Next week, I'll do the procedure. Take medicine on time).

Patient: Sahib, mainu bohat veer ho rahi si meri budhi nay mainu long dita ta kay aram aa jaway, par... (I was having a lot of pain. My wife gave me "long" for pain, but...)

Dentist (head down, sternly): mai nay jo dawa di ha aap os say theek ho jao gay inshallah. (The medicine that I have advised you will cure you, inshallah).

Another Patient: Mainu kab check karo gay do ghantay say baitha hoo... (When will be my turn? I am sitting for 2 hours.)

Dentist (to Nurse): Dusra patient tiyaar karain (get the other patient ready please)

Patient: bohat meharbani daktar sahib. Allah aapko khush rakhay. Rab day hawaly. (Thank you so much doctor,,,may Allah bless you).

\section{Solidarity is Power}

Considering the solidarity theme, $20 \%$ of the doctors and $30 \%$ of the patients believed that no power existed in doctor-patient interaction. They believed that power dynamics were irrelevant or a temporary relation. The doctors were aware of their position as the service providers and the patients' position as the service receivers. One of the doctors said: "What Power? Oh no, I do not think so...It is our moral duty not to impair their 
trust."However, there were only twodoctors who believed that the patients had equal power as they had. The participants indicated that power was reduced to none or power differentials between the doctors and the patients were dissolvedor inconsequential. A few of them believed in a friendly relationship with their patients. They did not provide an insight into power manifesting in the doctor-patient interactions (figure). They subconsciously relinquished power by including patients in the decision-making process such as in palliative care unit ${ }^{8}$, moderated it by letting the patients take their names and also by

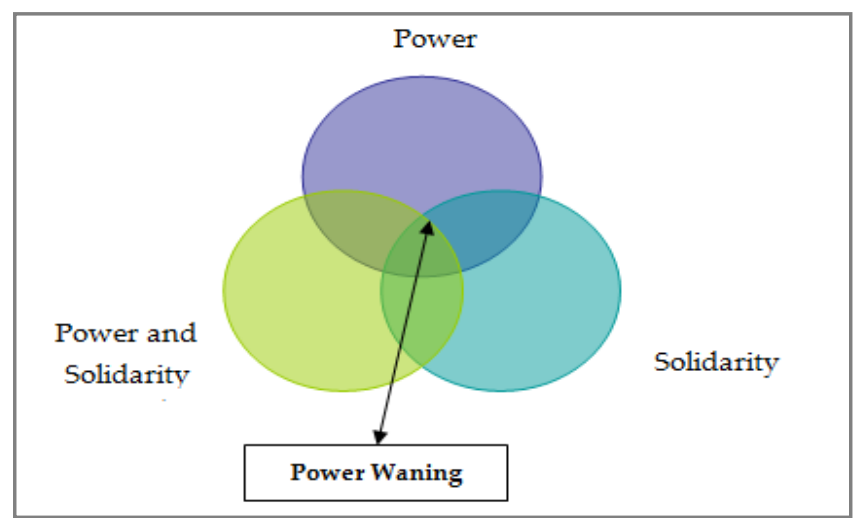

Figure-1: Power waning in medical consultations.

remembering the names of their patients (such as; "Ji Bibi Hajra") and by surrendering their authority and accepting when the patient no longer required or dropped their services.

The examples of doctors and patients showing complete solidarity were none in the observational data, despite the doctors and patients' perceptions of the need to have cooperation in doctor-patient interactions. The observations could not prove the perceptions of the doctors and patients about solidarity.

\section{DISCUSSION}

The research aimed to find perceptions of the doctors and patients about power-sharing in medical interactions. Contrary to the results of study by Nimmon et al , which used Bourdieu's framework to appropriate the social practice theory only to the theme of power, this study revealed that Bourdieu's concept of doxa and habitus is applicable to all the three emerging themes of the current study consistent with the previous studies by Penn et al, and Nomura et $a l$, this study also reveals that the doctors are unaware of power relations as their profession subconsciously empowers them ${ }^{9,10}$.

Various perceptions of doctors about power relations in this study are suggestive. For example, there may be other factors apart from their profession that interfere with their perceptions. As indexed by Kaba and Sooriakumaran,these factors may include the context, level of formality, experience, training, and lack of time ${ }^{11}$. Apart from these, the other personal factors found in our study are; beliefs, values, and preferences. Supported by Agyemang-Duah et al, our research suggests that the background, nature, and social set-up and the social circle of health-care professionals and patients contribute to developing their personality, and so, varying perceptions ${ }^{13}$. Considering the peripheral factors of their workplace as identified by Page et al, in UK, who found that the unequal positions of doctors, result in low professional pursuit levels. That is why some of them are considered more successful than the others, which empowers them to interfere in communication practices with their patients ${ }^{12}$. As the factors mentioned above shape an individual differently, their power relations with patients and their perceptions on it are also very different. As aligned with the results of a study by Nimmon, the observations of this study also unfolds that experienced doctors are more aware of the power dynamics ${ }^{8}$. The implications and insights of this research are drawn from each of the three themes.

Regarding the power theme, the doctors and the patients believed that the doctors were the sole owners of power. The data shows that the doctors reflected on how they managed and handled their power. They had internalized their habitus and were aware of the medical power available to them. Most of the experienced doctors believed that knowledge sharing empowered them. Approving the results from a study by Nimmon, this study also suggests that the doctors used language strategies to handle patients 
in line with Bourdieu's social theory, which states that language is a means of communication and is a medium of power8. The paternalistic relationship renders the role of an adult to a doctor and an infant to a patient. Like an infant, the patient is powerless and cannot participate in decisionmaking, while the doctor is all-powerful and autonomous ${ }^{15}$. Similar findings are replicated in this study; that power is relational, interpersonal, co-constructed, contextualized, and institutionalized ${ }^{14}$.

The theme of power and solidarity recorded that the power of doctorsis declining. In conformity with the findings of a study by Harzheim et al, the researchers found that patients are becoming autonomous and powerful because healthcare has become a commodity ${ }^{16}$. The doctors have assumed the role of producers while the patients are consumers. As part of their training, habitus, and education as cultural capital that doctors subconsciously acquire, they do not feel for patients' problems, pain, and misery. Consistent with the results Lor et al, the patients are given an independent choice to make decisions for themselves, and doctors provide a solution to the problem, the options available, and the odds, but not their recommendations unless asked ${ }^{17}$.

The doctors believe that under the slogans of consumerism, the rhetoric of egalitarian and patient-focused healthcare facilities has rendered the doctors realize power-sharing equally with the patients during the decision-making process. Now patients have equal rights to intervene and discuss freely about what they would do with their bodies. Doctors are deliberately giving up their power for material goods. Approving the results of a study by Chima in South Africa, this study shows that the patients or their relatives consider it their right to have full knowledge and a clear picture of their disease ${ }^{14}$, However, this power waning is subconscious and has its implications.

Considering the theme of solidarity, the participants believein the non-existence of power, both parties render each other equal and show solidarity towards each other. This relation is of mutual participation. The participants believe that mutual consensus between the two parties is of utmost importance for common benefit. For shared participation, it is important to have interaction based on equal power relations. It renders a great responsibility on the patient as he is fully responsible for his health decisions. The doctor's satisfaction is derived from the services he provides to humanity. The doctor strives to understand the patient's personality to provide a full understanding of his illness ${ }^{18}$. This relation is entirely different from the role of doctor being paternalistic, and can be compared to an adultto-adult relationship ${ }^{19}$. There is a shift from the paternalistic attitude to mutual participation, giving each party an equal power relation ${ }^{20}$. However, such behaviour was non-existent in the observational data, although this behaviour was verbally professed by both the doctors and the patients.

The research has some limitations. The findings may not be generalizable for the overall healthcare systems in Pakistan. Besides that, our research focused on doctor-patient encounters in out-patient departments only. This excludes data collection from emergency departments and inpatient wards. The future researchers may explore this area in the different kinds of public and private hospitals by including patients of diverse socio-economic and linguistic backgrounds; urban/rural, literate/ illiterate, and male/female to capture the full picture of power differentials.

\section{ACKNOWLEDGEMENT}

We wish to acknowledge the efforts of hospital personnel who provided us every facility in collecting data and supported us in performing our research activities.

\section{Funding Disclosure}

There is no funding source and financial sponsor for the design, execution, analysis, and interpretation of data or writing of this research study. No authority or person played any involvement or role in conducting this research work. 


\section{CONCLUSION}

The modern rhetoric focuses on patientcentered healthcare, rendering equal power relations between the doctors and patients, which are exercised by knowledge sharing, including the patients in decision-making processes, and improving communication skills. However, this concept is not fully realized in the Pakistani context. The doctors always have power available to them through their habitus and doxa, which gives them knowledge. The researchers conclude that those aware of power benefit, share, exert, and relinquish it moderately according to the situational context. Most of the patients recognize that doctors are the authorities who hold power. Doctors and patients need to internalize these rules of the game to benefit fully in their interactions. Doctors should improve their communication skills so they can handle their power efficiently.

\section{CONFLICT OF INTEREST}

This study has no conflict of interest to be declared by any author.

\section{REFERENCES}

1. Drinka T, Ray RO. An investigation of power in an interdisciplinary health care team. Gerontol Geriatr Educ 1987; 6(3): 43-53.

2. Bourdieu P. Language and symbolic power. Harvard University Press;1991https://books.google.com.pk/books?id= u2ZlGBiJntAC\&printsec $=$ frontcover\&dq $=2 . \% 09$ Bourdieu + P. + Language+and+symbolic+power.+Harvard+University+Press;+ 1991\&hl=en\&sa $=$ X\&ved=2ahUKEwikja_8u53tAhWeUxUIHRtiD 04Q6AEwAHoECAIQAg\#v=onepage\&q=2.\%09Bourdieu $\% 20 \mathrm{P}$. $\% 20$ Language $\% 20$ and $\% 20$ symbolic $\%$ 20power. $\% 20$ Harvard $\% 20$ University \%20Press \%3B\%201991\&f=false.

3. Sun N, Rau P-LP. Barriers to improve physician-patient communication in a primary care setting: perspectives of Chinese physicians. Int J Behav Med 2017; 5(1): 166-176.

4. Nimmon LE. Meaning making within the social activity domain of health maintenance: the role of social networks [PhD Thesis]. University of British Columbia; 2014 [Internet]. https://open. library.ubc.ca/cIRcle/collections/ubctheses/24/items/1.01670.

5. Bourdieu P. Distinction: A social critique of the judgement of taste. Harvard University Press; 1984; 306(1): 484-91.

6. Fairclough N. Critical discourse analysis: The critical study of language. Routledge; 2013 [Internet]. https:/ / books.google.com. $\mathrm{pk} /$ books?hl=en\&lr=\&id=nf7cAAAAQBAJ\&oi=fnd\&pg=PP1\&d q=Fairclough $+\mathrm{N} .+$ Critical+discourse+analysis:+The+critical+stu
dy+of+language.+Routledge \%3B+2013+Sep+13.\&ots=15QfOqtO XJ\&sig=-X52TrjKnrKTEEja1OfD97WViys\&redir_esc=y\#v= onepage\&q\&f=false.

7. Bélanger E, Rodríguez C, Groleau D, Légaré F, Macdonald ME, Marchand R. Initiating decision-making conversations in palliative care: an ethnographic discourse analysis. BMC palliat care 2014; 13(1): 63-74.

8. Nimmon L, Stenfors-Hayes T. The "Handling" of power in the physician-patient encounter: perceptions from experienced physicians. BMC Med Educ 2016; 16(1): 114-20.

9. Penn C, Watermeyer J. Verbal and non-verbal dimensions of the intercultural health setting. In: communicating across cultures and languages in the health care setting. Springer 2018; 1(2): 207-61.

10. Nomura K, Yamazaki Y, Gruppen LD, Horie S, Takeuchi M, Illing J. The difficulty of professional continuation among female doctors in Japan: a qualitative study of alumnae of 13 medical schools in Japan. BMJ Open 2015; 5(3): e005845.

11. Kaba R, Sooriakumaran P. The evolution of the doctor-patient relationship. Int J Surg 2007; 5(1): 57-65.

12. Page VJ, Ely EW. Delirium in critical care. Cambridge University Press; 2015 https://books.google.com.pk/books?hl=en\&lr=\& $\mathrm{id}=8 \mathrm{bDGBgAAQBAJ} \&$ oi $=$ fnd \&pg $=$ PR9\&dq $=$ Page $+\mathrm{VJ},+\mathrm{Ely}+\mathrm{E}$ $\mathrm{W} .+$ Delirium+in+critical+care.+Cambridge+University+Press $\%$ $3 \mathrm{~B}+2015 .+$ \&ots $=r-E 6 x Y$ PsKF\&sig $=8 \mathrm{mMKVCu}$ T $x n 4 \mathrm{swfi}$ 7111KzpI08A\&redir_esc $=y \# v=$ onepage\&q\&f=false.

13. Agyemang-Duah $\bar{W}$, Arthur-Holmes F, Peprah C, Adei D, Peprah P. Dynamics of health information-seeking behaviour among older adults with very low incomes in Ghana: a qualitative study. BMC Public Health 2020; 20(1): 1-3.

14. Chima SC. "Because I want to be informed, to be part of the decision-making": Patients' insights on informed consent practices by healthcare professionals in South Africa. Niger J Clin Pract 2015; 18(7): 46-48.

15. Kirby E, Lwin Z, Kenny K, Broom A, Birman H, Good P. “It doesn't exist": negotiating palliative care from a culturally and linguistically diverse patient and caregiver perspective. BMC Palliat Care 2018; 17(1): 90-98.

16. Harzheim L, Lorke M, Woopen C, Jünger S. Health Literacy as Communicative Action-A Qualitative Study among Persons at Risk in the Context of Predictive and Preventive Medicine. Int J Environ Res Public Health 2020; 17(5): 1718-44.

17. Lor M, Martinez GA. Scoping review: definitions and outcomes of patient-provider language concordance in health. Patient Educ Couns 2020; 103(10): 1883-01.

18. Marmorat T, Rioufol C, Ranchon F, Préau M. Encounters between medical and lay knowledge in therapeutic patient education. A qualitative study based on an oral chemotherapy program. Patient Educ Couns 2020; 103(3): 537-43.

19. Miao M, Dahm MR, Li J, Thomas J, Georgiou A. Managing uncertainty during the communication of diagnostic test information between patients and clinicians in australian emergency care. Int J Qual Stud Health Well-being 2020: 1049732320913037.

20. Pun JK, Chan EA, Wang S, Slade D. Health professional-patient communication practices in East Asia: An integrative review of an emerging field of research and practice in Hong Kong, South Korea, Japan, Taiwan, and Mainland China. Patient Educ Couns 2018; 101(7): 1193-06. 